\title{
Corrigendum to "A Novel MILP Model for the Production, Lot Sizing, and Scheduling of Automotive Plastic Components on Parallel Flexible Injection Machines with Setup Common Operators"
}

\author{
Beatriz Andres $(\mathbb{D}$, Eduardo Guzman $\mathbb{D}$, and Raul Poler $\mathbb{D}$ \\ Research Centre on Production Management and Engineering (CIGIP), Universitat Politècnica de València (UPV), \\ Calle Alarcón 03801 Alcoy, Alicante, Spain \\ Correspondence should be addressed to Beatriz Andres; bandres@cigip.upv.es
}

Received 5 August 2021; Accepted 5 August 2021; Published 18 November 2021

Copyright (c) 2021 Beatriz Andres et al. This is an open access article distributed under the Creative Commons Attribution License, which permits unrestricted use, distribution, and reproduction in any medium, provided the original work is properly cited.

In the article titled "A Novel MILP Model for the Production, Lot Sizing, and Scheduling of Automotive Plastic Components on Parallel Flexible Injection Machines with Setup Common Operators" [1], the authors identified errors in the datasets used in the study. Specifically, there was an error in the $c i$ (inventory cost) parameter which uses decimal values; however, the python code used it as an integer. The datasets have therefore been corrected in Tables 6-11, and the corresponding sections in the results section have been updated to reflect this. A revised data availability statement has also been provided. The authors confirm that the results and conclusions are the same as reported in the original article despite the revised datasets.

The corrected article is as follows:

In this article, a mixed integer linear program (MILP) model is proposed for the production, lot sizing, and scheduling of automotive plastic components to minimize the setup, inventory, stockout, and backorder costs, by taking into account injection molds as the main index to schedule on parallel flexible injection machines. The proposed MILP considers the minimum and maximum inventory capacities and penalizes stockout. A relevant characteristic of the modeled problem is the dependence between mold setups to produce plastic components. The lot-sizing and scheduling problem solution results in the assignment of molds to machines during a specific time period and in the calculation of the number of components to be produced, which is often called lot size, following a sequence-dependent setup time. Depending on the machine on which the mold is setup, the number of units to be produced will be distinct because machines differ from one another. The stock coverage, defined in demand days, is also included in the MILP to avoid backorders, which is highly penalized in the automotive supply chain. Added to this, the proposed model is extended by considering setup common operators to respond to and fulfill the constraints that appear in automotive plastic enterprises. In this regard, the MILP presented solves a lot-sizing and scheduling problem, emerged in a second-tier supplier of a real automotive supply chain. Finally, this article validates the MILP by performing experiments with different sized instances, including small, medium, and large. The large-sized dataset is characterized by replicating the amount of data used in the real enterprise, which is the object of this study. The goodness of the model is evaluated with the computational time and the deviation of the obtained results as regards the optimal solution.

\section{Introduction}

Production planning, sequencing, and scheduling are key operations performed by enterprises, and any circumstances or events that affect them strongly influence the supply chain operation in which they are embedded. All these three 
planning levels are characterized by the decision-making time horizon in accordance with three decision-making levels: strategical, tactical, and operational. Thus, production planning is set at the strategic level by considering families of products, while sequencing and scheduling are set at a more operational decision-making level. Accordingly, Gujjula et al. [1] proposes the following differentiation between these two concepts: (i) production scheduling deals with the assignment of production orders to production intervals with a short planning horizon and specific time periods and (ii) production sequencing deals with the sequence of production orders for each production interval.

Our aim is to solve a lot-sizing and scheduling problem with a sequence-dependent setup on parallel flexible machines. To this end, a mixed integer linear program (MILP) model is proposed to minimize the setup, inventory, stockout, and backorder costs by taking into account injection molds as the main index to schedule on parallel flexible injection machines. We also consider setup common operators to extend the proposed basic MILP model.

This article focuses on the specific production lot-sizing and scheduling problem (LSSP) in parallel flexible machines. According to Kim et al. [2], the LSSP deals with the minimization of production and inventory costs by simultaneously optimizing lot sizes and production schedule. The authors also refer to small and big bucket models depending on the number of allowed setups. Accordingly, production scheduling assigns production orders to production intervals with a short-term planning horizon lasting several days or shifts. Moreover, the assignment in the scheduling process has an implicit sequence for each production shift. Therefore, the main goal is to identify the time period when to produce, the quantity to produce as the lot size in units or timeslots, and the production sequence required to meet demand and to avoid backorders and stockouts.

As enterprises are seen as complex systems in operations management, their operation mechanisms are difficult to manage. This difficulty further increases because enterprises belong to a supply network system, in which the complexity and relationships with external actors are latent. Current global market conditions and constant changes in the supply chain environment render enterprises as complex systems. Moreover, researchers have to bear in mind that market consumers are currently used to acquire highly personalized products, which is known as mass customization with short development periods ("time to market").

In the last few years, novel technologies have been increasingly used, such as cloud computing, big data, artificial intelligence, and machine learning. This has become a trend that has boosted companies to transform their way of operating at enterprise and supply chain levels. The result of this digital transformation has been coined as Industry 4.0 [3]. Nevertheless, the application of Industry 4.0 to smalland medium-sized enterprises (SMEs) is not as idealistic as the Industry 4.0 definition indicates. The literature includes different studies on implementing Industry 4.0 technologies into different sectors. The present work focuses on the literature review by Echchakoui and Barka [4], which studies the impact that Industry 4.0 has on the plastics industry. The relevance of the work by Echchakoui and Barka [4] is aligned with the present article because we develop MILP to support the LSSP in a company that belongs to the plastic sector by particularly focusing on the injection of plastic automotive components. In the aforementioned literature review, the authors highlight that implementing Industry 4.0 research into the plastics industry is still in its initial stages, but research in this field is growing.

The origin of this article lies in the H2020 Project Cloud Collaborative Manufacturing Networks (C2NET) [5], whose research focuses on providing a cloud platform, in which a set of tools and technologies are embedded to support the following: (i) data management and interoperability; (ii) the optimization of plans at enterprise and supply chain levels; and (iii) the integration of collaborative processes among supply network members. The C2NET cloud platform is built according to an open-source philosophy, which makes it affordable and easy to use by SMEs in terms of both monetary and expertise or knowledge required for its use. The optimization module contains advanced optimization models and algorithms to support and calculate replenishment, production, and delivery plans at both the enterprise and network levels. The calculation of plans at the enterprise level is characterized by only considering the resources and data from a single enterprise, while the calculation of plans at the network level uses the constraints, resources, and data from two supply chain enterprises, or more, as input data. When solving replenishment, production, and delivery plans from a collaborative network perspective, the output data of one enterprise plan are used as the input data of another enterprise plan. If we consider two enterprises from a network, $A$ and $B$, where $A$ is the supplier and $B$ is the manufacturer, the replenishment plan of company B is constrained by the production plan of company A. Hence, information is exchanged and a loop plan is calculated until the materials required by company $B$ coincide with the materials that can be produced by company A. On the C2NET cloud platform (CPL), the negotiation loop is operated by the collaborative module (COT), the plan calculation is managed by the optimization module (OPT), and data exchange and information interoperability are handled by the data collection framework module (DCF) [6]. The operation and management of the complex large-scale systems that characterize enterprises are covered by the models and tools developed in the CLP with the help of embedded intelligent methods.

Different enterprises from diverse sectors were involved in the C2NET project to validate and test the generated results. The enterprise study object of this article is the automotive industry, which is included in one of the industrial pilots. The automotive supply chain is characterized by the need to perform flexible manufacturing to meet the demand of the original equipment manufacturer (OEM) in terms of delivery time, scheduling, and lot size in a just-intime (JIT) production system [7]. When addressing the LSSP in the automotive industry, suppliers are highly penalized when the components supplied to the OEM are delayed. 
Thus, the automotive components' manufacturers have to manage minimum and coverage stocks to avoid potential penalizations. The coverage goal is set in demand days so that suppliers have to produce at least 3 days of demand in advance (commonly used in the automotive industry) to stock production, as advances to avoid delaying demand because it could imply stopping the automotive assembly line in OEM facilities.

The main objective of this article is to model a real problem from a second-tier supplier in the automotive supply chain and to solve it in a reasonable computation time. Accordingly, a lot-sizing and sequencing model is hereafter provided to respond to the requirements of a second-tier supplier from an automotive supply chain. The proposed optimization model is embedded in the OPT module of the C2NET CPL. A novel MILP model for automotive plastic components' production lot-sizing and scheduling on parallel flexible injection machines is proposed with setup common operators. The MILP model was implemented in Pyomo [9], used as an extensible python-based open-source optimization modeling language for linear programming and nonlinear programming, among others. Finally, a complex largescale problem is addressed to deal with the scheduling plan of an automotive components' manufacturer.

The reminder of this document is organized as follows. Section 2 presents a literature review to gain insight into works that have addressed similar type problems and to justify this study's contribution. Section 3 describes and analyzes the problem to be solved. Section 4 presents the novel MILP model for the automotive plastic components' production lot-sizing and scheduling on parallel flexible injection machines. We take this MILP as the base model. Section 5 puts forward an extension of the base model, which contemplates common setup operators in the proposed LSSP to provide a more realistic perspective to the base model by taking into account the studied plastic components' injection industry. Section 6 offers comprehensive numerical experimentation by considering different data sizes for both proposed models, including small, medium, and large datasets. This article is concluded by discussing the findings obtained with the addressed problem.

\section{Related Studies}

A large amount of research has been conducted into different LSSP characteristics, but very few studies present optimization approaches that combine or integrate LSSP characteristics, that is, lot sizing and scheduling. According to Ríos-Solís et al. [10], the product-part-mold-machine (PPMM) problem and the part-mold-machine (PMM) problem study this combination of approaches and indicate that this problem type is scarcely addressed in the literature. Ríos-Solís et al. [10] defined products as final products and parts as components that derive from the bill-of-materials parts explosion. Furthermore, Ríos-Solís et al. [10] classified the PPMM as bilevel capitated LSSP. Accordingly, the first part of the PPMM and PMM lot-sizing and scheduling problems seeks to determine the optimal size of either a lot of products in the PPMM approach or the parts in the PMM approach. Both approaches seek to assign parts or pieces to molds and molds to the machines. The second part is involved in the scheduling that aims to determine the order of processing molds in machines during each planning time period.

Studies such as Ibarra-Rojas et al. [11] addressed the problem of manufacturing parts that are produced in molds and are mounted on machines. This study proposes an MILP that seeks to maximize the production of parts and also determines the batch size of every part and the assignments of parts to mold and machines. An update of this study is that proposed by Ríos-Solís et al. [10], which aims to determine the lot size of a finished product; that is, it determines the number of finished products to be manufactured, the number of parts to be manufactured, and the assignment of the parts to the mold and the mold to the machine. The model of Ríos-Solís et al. [10] also contemplates the feasibility of scheduling molds in machines during each period. However, in their experimental results, they only handle one period of time periods and propose working with multiple time periods as a future research line. Different approaches have been proposed in the literature to optimally solve scheduling in engineering; in this regard, $\mathrm{Li}$ et al. [12] highlighted the importance on the use and application of MILP to deal with the aforementioned problem in the engineering research area.

Studies about LSSP are paid more attention by researchers and companies given their applicability to the real world [13]. In our literature review, we find studies that integrate batch sizing decisions into restrictions for scheduling problems (see Table 1), studies such as that presented by Stadtler [14], in which a combined approach to support a single machine LSSP is proposed. For this purpose, an MILP is formulated to minimize maintenance and inventory setup costs over a planning horizon. Wolosewicz et al. [15] combined production planning and scheduling by proposing an MILP that seeks to determine the lot size for a fixed sequence of operations in the machines, taking into account the times and operating costs. Kim et al. [2] presented a combined approach in an MILP that seeks to minimize the sum of the cost of production, installation, and inventories. James and Almada-Lobo [16] presented an MILP for the scheduling problem and capacitated lot-sizing of a single machine and a parallel machine with sequence-dependent setup times and costs. Meyr and Mann [17] put forward an MILP to simultaneously determine production lot sizes and schedules on nonidentical parallel production lines.

Our model deals with the problem contemplated by Ríos-Solís et al. [10] and Ibarra-Rojas et al. [11]. By continuing with the future research lines indicated by these authors, our study considers many periods when modeling and running experiments to solve the LSSP. The base model herein proposed also bears in mind stock coverage constraints, which are typical in the studied automotive supply chain industry context. The proposed novel MILP also contemplates an objective function 
TABLE 1: Literature review of recent lot-sizing and scheduling problems.

\begin{tabular}{|c|c|c|c|c|}
\hline Authors & Objectives & Industrial application & Resolution methods & Solver \\
\hline [2] & $\begin{array}{l}\text { Minimize the sum of production, setup, and } \\
\text { inventory costs }\end{array}$ & Zinc refinery & $\begin{array}{l}\text { Heuristic algorithm combining a } \\
\text { decomposition scheme with a local } \\
\text { search procedure }\end{array}$ & CPLEX \\
\hline [11] & Maximize the weighted cost of produced pieces & $\begin{array}{l}\text { Automotive, } \\
\text { consumer goods, and } \\
\text { toys }\end{array}$ & Decomposition approach & $\begin{array}{l}\text { CPLEX } \\
11.2\end{array}$ \\
\hline [14] & $\begin{array}{l}\text { Minimize the sum of inventory holding and setup } \\
\text { costs in the planning interval }\end{array}$ & Pharmaceutical & & $\begin{array}{l}\text { Xpress- } \\
\text { MP }\end{array}$ \\
\hline [10] & Maximize the profit of finished products & $\begin{array}{l}\text { Automotive, } \\
\text { consumer goods, and } \\
\text { toys }\end{array}$ & $\begin{array}{l}\text { Iterative heuristic based on } \\
\text { mathematical programming }\end{array}$ & $\begin{array}{c}\text { Gurobi } \\
6.05\end{array}$ \\
\hline [17] & $\begin{array}{l}\text { Minimize inventory holding, sequence-dependent } \\
\text { setup, and line-specific production costs of } \\
\text { potentially heterogeneous production lines }\end{array}$ & 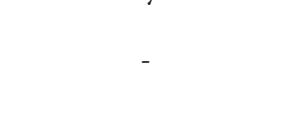 & Decomposition-based approach & $\begin{array}{c}\text { GLPK } \\
4.44\end{array}$ \\
\hline [15] & $\begin{array}{l}\text { Minimize the sum of production, inventory and } \\
\text { setup costs }\end{array}$ & - & Lagrangian heuristic & $\begin{array}{l}\text { Xpress- } \\
\text { MP }\end{array}$ \\
\hline
\end{tabular}

based on the assembly line and allows idle times among molds, which is a fundamental characteristic for real cases and has been ignored by former studies. Finally, the base model is extended by offering a scenario that comes closer to reality by considering another index that represents the workers who change molds and, thus, bears in mind the casuistry associated with the LSSP that refers to the setups of the usual operators.

\section{Problem Description}

The main aim of this article is to propose a novel MILP model for the production scheduling carried out by an automotive plastic components' manufacturer that acts as a second-tier supplier in the automotive supply chain. Plastic components are produced in molds that are mounted on parallel flexible injection machines. Injection machines shape plastic pellets into automotive semifinished products to then be assembled on an OEM production line. Nevertheless, for industry, studying car components is treated as the second-tier supplier's end products.

According to Ríos-Solís et al. [10], lot-sizing and scheduling plans of mold-injection enterprises entail decision-making to determine (i) the lot size as the amount of components to be produced during a period of time or a number of periods to produce the same component and (ii) the assignment of molds to machines by considering that components can be produced only in specific molds with their shape cavity.

The second-tier supplier herein studied is characterized by having specific molds that produce each automotive component. When two molds are available to produce the same component, these molds involve different processing times given their technical particularities. Each mold can also be setup on different machines to produce the same automotive component, but the same mold mounted on different machines has different production rates depending on the machine on which it is setup. Accordingly, productivity differs depending on the mold and machine assignment (see Figure 1). Moreover, in the automotive sector, two different parts are produced in the same mold, including right- and left-hand parts, which is known as biproduct injection molding [18].

The studied enterprise has 21 injection machines and a set of molds to produce the range of components delivered to the first-tier supplier and finally to the various OEMs that belong to different automotive supply chains, depending on the car brand. The enterprise works three shifts per day over a 5-day week and reserves overtime shifts on the sixth weekday in the event of production not finishing during the normal operation time. Workers are told that they will work overtime periods at least 1 week beforehand.

Molds are changed using cranes and, therefore, a setup time is incurred. A limited number of workers are in charge of changing and mounting molds to the assigned machines. Moreover, these workers, known as setup common operators, work only two of the three shifts that the enterprise arranges.

One of the requirements of the enterprise's study is that, once the mold is setup on a machine, the mold should remain for at least $24 \mathrm{~h}$ so as not to saturate the work of the limited setup common operators and to not incur on too many setups because the setup time is estimated between 1 and $3 \mathrm{~h}$, which obviously has an associated setup cost. If a longer production time is needed, the mold is set up during the required time periods without incurring any setup costs. Thus, the modeled problem should contemplate no setup carry-over cases.

Backorders are highly penalized in the automotive supply chain. Therefore, enterprises in the automotive industry use stock coverage, which indicates the number of demand days that stocks can cover. Normally in the automotive industry, stock coverage is set at 3 demand days; for example, for the demand of four units for the next three periods ( $d_{1}=4$ units, $\mathrm{d}_{2}=4$ units, and $\mathrm{d}_{3}=4$ units), stock coverage is defined as 12 units at the end of the first period. Moreover, as warehouses have space limitations, a maximum inventory is considered.

With regard to the demand, the automotive industry updates the demand during each period for the next five 


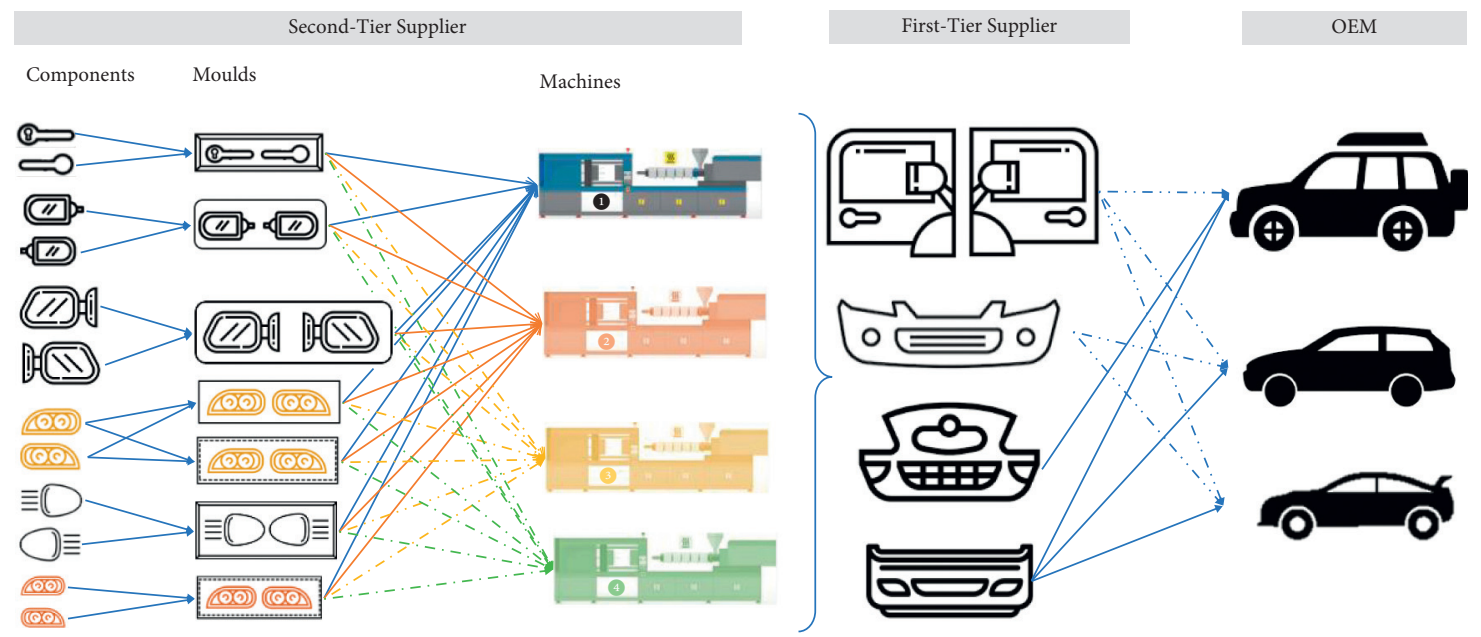

Figure 1: Production scheme on parallel flexible injection machines of plastic components in the automotive supply chain.

frozen periods. The OEM works with considerable demand information (1 year of demand horizon), but only communicates the demand for the next 6 months to the first-tier supplier. Finally, first- and second-tier suppliers normally work to a 3-month demand horizon and with daily periods. The LSSP considers a 21-day horizon.

A detailed flowchart of the processes is presented in Figure 2, from the generation of the customer demand to the final resolution of the second-tier supplier model. Accordingly, in the automotive supply chain, the OEM generates customer orders, according to the final customer demand. The OEM transforms the customer orders into the master production scheduling (MPS), which jointly with bill of materials (BOM) and the inventory availability computes the materials requirement plan (MRP). The OEM demand plan is generated from the MRP and transferred to the first-tier supplier. Then, the first-tier supplier obtains its demand by using a simple bill-of-materials parts explosion given the OEM demand plan. The second-tier supplier estimates the component requirements from the final requirements in a frozen sequence transferred by the first-tier supplier, and the demand plan is generated [8]. Then, the second-tier supplier proceeds to compute the LSSP model with the aim of (i) assigning molds to machines, (ii) scheduling the processing molds in machines during each planning time period, and (iii) calculating the optimal lot size of products. The LSSP is modeled by considering molds to be the main index. The proposed production lot-sizing and scheduling base model has as main inputs the data parameters described in Table 2. Nevertheless, if setup common operators are considered when solving the
LSSP, data parameters related with the type of operators have to be taken into account for building the extended version of the base model. Finally, the proposed MILP is solved by the second-tier supplier using the Gurobi solver obtaining optimal or near-optimal solutions of the production LSSP. In a nutshell, the objective of the proposed MILP is to minimize the total costs, namely, setup and inventory costs, penalization costs for coverage stockouts, backorder costs, and tool setup costs, such as the route cost of selecting one machine or another to setup a tool.

\section{MILP Model for Lot-Sizing and Scheduling on Parallel Flexible Injection Machines: Notation and Model Formulation}

The lot-sizing and scheduling on parallel flexible injection machines problem under study is notated in Table 2, where the main indexes related to machines, tools, products, and periods are notated. The input data parameters are presented, and decision variables are established as the output data of the MILP model for lot-sizing and scheduling on parallel flexible injection machines.

The formulation of the MILP model for lot-sizing and scheduling on parallel flexible injection machines is described next. The objective function minimizes total costs, including setup and inventory costs, penalization costs for coverage stockouts, backorder costs, and tool setup costs, such as the route cost of selecting one machine or another to set up a tool.

$$
\operatorname{Min} z=\sum_{i} \sum_{j} \sum_{t} c s_{j} \cdot S A_{i j t}+\sum_{k} \sum_{t} c i_{k} \cdot \mathrm{INV}_{k t}+\sum_{k} \sum_{t} c s t_{k} \cdot S T_{k t}+\sum_{k} \sum_{t} c b_{k} \cdot B_{i t}+\sum_{i} \sum_{j} \sum_{t} r_{i j} \cdot c r_{i j} \cdot S A_{i j t},
$$




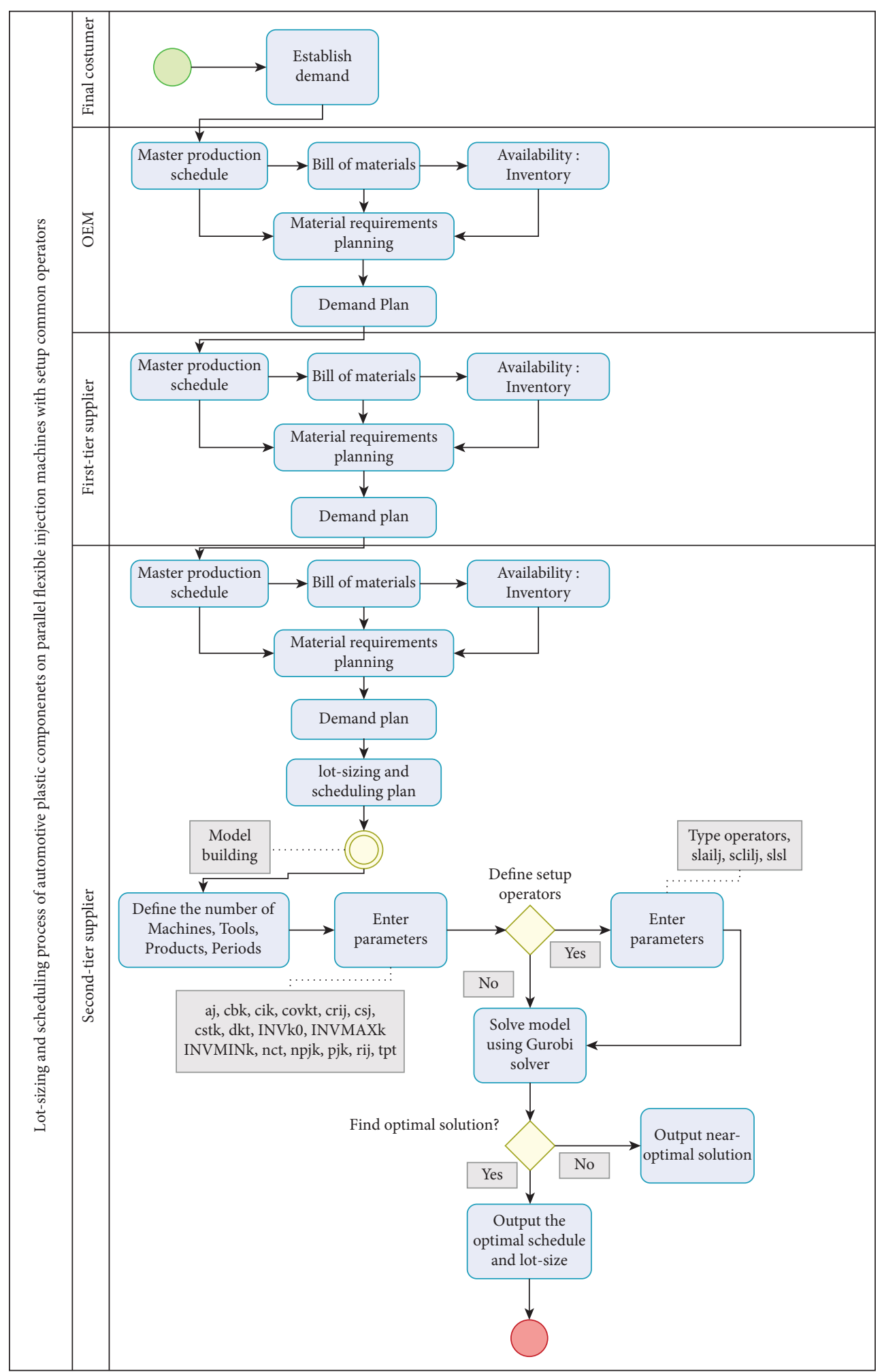

FIgURE 2: Flowchart of the proposed solution methodology. 
TABLE 2: Nomenclature for the model.

\begin{tabular}{|c|c|}
\hline \multicolumn{2}{|r|}{ Index } \\
\hline $\bar{i}$ & Index of machines $i \in\{1, \ldots, I\}$ \\
\hline$j$ & Index of tools $j \in\{1, \ldots, J\}$ \\
\hline$k$ & Index of products (parts) $k \in\{1, \ldots, K\}$ \\
\hline$t$ & Index of time periods $t \in\{1, \ldots, T\}$ \\
\hline & Data \\
\hline$a_{j}$ & Total amount of tools $j$ available for production \\
\hline$c b_{k}$ & Backorder cost of product $k$. \\
\hline$c i_{k}^{n}$ & Inventory cost of product $k$ \\
\hline $\operatorname{cov}_{k t}$ & Stock coverage defined as the number of time periods for the stock minimum coverage of product $k$ during time period $t$ \\
\hline$c r_{i j}$ & Setup cost of tool $j$ on machine $i$ \\
\hline$c s_{j}$ & Setup cost of preparing tool $j$ \\
\hline$c s t_{k}$ & Coverage stockout cost of product $k$. \\
\hline$d_{k t}$ & Demand of product $k$ during time period $\mathrm{t}$ \\
\hline $\mathrm{INV}_{k 0}$ & Initial inventory of product $k$ \\
\hline INVMAX $_{k}$ & Maximum inventory units for product $k$ during time period $t$ \\
\hline $\operatorname{INVMIN}_{k}$ & Minimum inventory units for product $k$ during time period $t$ \\
\hline$n c_{t}$ & Amount of tool changes allowed during time period $t$ \\
\hline$n p_{j k}$ & Amount of products $k$ no longer produced when tool $j$ is set up \\
\hline$p_{j k}$ & Amount of products $k$ produced when tool $j$ is set up \\
\hline$r_{i j}$ & 1 if tool $j$ can be set up on machine $i, 0$ otherwise. \\
\hline$t_{p t}$ & $\begin{array}{l}\text { Production time available during time period } t \\
\text { Decision variables }\end{array}$ \\
\hline$B_{k t}$ & Backorder of product $k$ during time period $t$ \\
\hline $\mathrm{INV}_{k t}$ & Inventory level of product $k$ at the end of time period $t$ \\
\hline$S A_{i j t}$ & $\begin{array}{c}1 \text { if tool } j \text { is set up on machine } i \text { during time period } t \text { and is not set up on machine } i \text { during time period } t-1 ; 0 \text { if tool } j \text { is set up } \\
\text { on machine } i \text { during time period } t-1\end{array}$ \\
\hline$S_{i j t}$ & 1 if tool $j$ is set up on machine $i$ during time period $t, 0$ otherwise \\
\hline$S T_{k t}$ & Coverage stockout of product $k$ during time period $t$ \\
\hline$T P_{i j t}^{n t}$ & Production time of tool $j$ set up on machine $i$ during time period $t$ \\
\hline$X_{k t}$ & Amount of product $k$ to produce during time period $t$ \\
\hline$X n p_{k t}$ & Amount of product $k$ no longer produced during time period $t$, while a tool is set up \\
\hline$X_{k t}^{\prime}$ & Amount of product $k$ to produce during time period $t$ by subtracting $X n p_{k t}$ \\
\hline
\end{tabular}

subject to sequence constraints:

$$
\begin{aligned}
S_{i j t} \leq r_{i j}, \quad \forall i, j, t, \\
S A_{i j t} \leq r_{i j}, \quad \forall i, j, t, \\
\sum_{j} S_{i j t} \cdot r_{i j} \leq 1, \quad \forall i, t, \\
\sum_{i} S_{i j t} \cdot r_{i j} \leq a_{j}, \quad \forall j, t .
\end{aligned}
$$

Constraints (2) and (3) constrain only setup tools $j$ on previously assigned specific machines $i$. Constraint (4) determines that one or any tool $j$ can be set up for production during each time period $t$. Constraint (5) guarantees that the total amount of tools $j$ available for production can only be set up as a maximum during each time period $t$.

Production and capacity constraints:

$$
\begin{aligned}
X_{k t}< & =\sum_{i} \sum_{j} p_{k t} \cdot r_{i j} \cdot T P_{i j t}, \quad \forall k, t, \\
X n p_{k t}< & =\sum_{j} \sum_{i} n p_{j k} \cdot r_{i j} \cdot S A_{i j k}, \quad \forall k, t,
\end{aligned}
$$

$$
\begin{gathered}
X_{k t}^{\prime}=X_{k t}-X n p_{k t}, \quad \forall k, t, \\
T P_{i j t}=t p_{t} \cdot S_{i j t}, \quad \forall i, j, t .
\end{gathered}
$$

Constraint (6) computes the amount of product $k$ produced during time period $t$ and ensures that a specific tool $j$ is able to be set up on machine $i$ during time period $t$ when product $k$ is produced. Constraint (7) determines the amount of product $k$ no longer produced when tool $j$ is set up during time period $t$ on machine $i$ by considering that another tool $j$ is set up on machine $i$ during time period $t-1$. This also ensures that such a specific tool $j$ is able to be set up on machine $i$ during time period $t$ when product $k$ is produced. Constraint (8) computes the amount of product $k$ to be produced during time period $t$ by subtracting $X n p_{k t}$ as the amount of product $k$ no longer produced when tool $j$ is set up. Constraint (9) determines the production time used during time period $t$ when tool $j$ is set up on machine $i$. In $T P_{i j t}$, we indicate the production lot size during period times, e.g., 24 h. This means that if tool $j$ is set up on machine $i$, the tool cannot be changed by another one for the next $24 \mathrm{~h}$. Therefore, the minimum lot size corresponds to the products produced during the $24 \mathrm{~h}$ that tool $j$ is set up on machine $i$.

Setup constraints: 


$$
\begin{aligned}
S A_{i j t} & =S_{i j t}, \quad \forall i, j, t=1 \\
S A_{i j t} & \geq S_{i j t}-S_{i j t-1}, \quad \forall i, j, t>1, \\
S A_{i j t} & \leq 1, \quad \forall i, j, t>1, \\
\sum_{i} \sum_{j} S A_{i j t} & \leq n c, \quad \forall t .
\end{aligned}
$$

Constraint (10) allows the first setup of tool $j$ to be determined on machine $i$, which enables it to be modeled if tool $j$ is set up during time period $t$ on machine $i$ for the first time and decision variables $S_{i j t}$ and $S A_{i j t}$ take the same value 1. Constraint (11) ensures that $S A_{i j t}$ does not take values above 1. Constraint (12) limits the amount of tool changes allowed during time period $t$.

Inventory balance equations:

$$
\begin{aligned}
& \mathrm{INV}_{k t}=\mathrm{INV}_{i 0}+X_{k t}^{\prime}-d_{k t}+B_{k t}, \quad \forall k, t=1, \\
& \mathrm{INV}_{k t}=\mathrm{INV}_{k t-1}+X_{k t}^{\prime}-d_{k t}+B_{k t}-B_{k t-1}, \quad \forall k, t>1
\end{aligned}
$$

Inventory balance equations (13a) and (13b) guarantee appropriate values for the inventories, quantities to produce, and backorders for each time period $t=1$ and $t>1$, respectively.

Stock coverage constraint:

$$
\begin{gathered}
\mathrm{INV}_{k t} \geq \operatorname{INVMIN}_{k t}, \quad \forall k, t, \\
\mathrm{INV}_{k t} \leq \mathrm{INVMAX}_{k t}, \quad \forall k, t, \\
\mathrm{INV}_{k t}+S T_{k t} \geq \sum_{c=1}^{c=c o v} \mathrm{~d}_{k(t+c)}, \quad \forall k, t<\mathrm{T}-\operatorname{cov} .
\end{gathered}
$$

Constraints (14) and (15) limit the inventory levels for each product $k$ according to the available space for inventory holding during time period $t$. Constraint (16) is a constraint for the stock coverage of products.

Bound and nature variables:

$$
\begin{gathered}
S A_{i j t}, S_{i j t} \in\{0,1\}, \quad \forall i, j, t, \\
X_{k t}, \mathrm{INV}_{k t}, B_{k t}, S T_{k t}, X n p_{k t}, B X_{k t}^{\prime}, \in \mathbb{N}, \quad \forall k, t, \\
T P_{i j t} \in \mathbb{N}, \quad \forall i, j, t .
\end{gathered}
$$

Constraint (17) indicates the binary nature of setup $S_{i j t}$ and the setup amount $S A_{i j t}$ variables. Constraints (18) and
(19) indicate the continuous nature of the represented variables.

\section{MILP Model for Lot-Sizing and Scheduling on Parallel Flexible Injection Machines with Setup Common Operators: Notation and Model Formulation}

The MILP model for lot-sizing and scheduling on parallel flexible injection machines described in the above section provides a solution to an LSSP that emerged in a second-tier supplier of a real automotive supply chain. In this section, the base model is extended by considering setup common operators to respond and fulfill the constraints that arise in automotive plastic enterprises. In this regard, the base model is extended to offer coming closer to reality by considering another index which represents the workers who change molds. It also bears in mind the casuistry associated with the LSSP problem that refers to setup common operators.

The nomenclature for the extended model is represented in Table 3. In order to avoid repetitions, the data and decision variables used for the MILP model for lot-sizing and scheduling on parallel flexible injection machines with setup common operators are considered to be the same as in the base model. Table 3 only shows the new data and decision variables in relation to the previous base model. There are different types of operators and a distinct number of workers corresponding to each operator type. For example, let us consider only one setup operator type that corresponds to the qualified technician category and is specialized in changing molds. Companies have a setup operator type corresponding to the auxiliary technician category, whose task involves helping the qualified technician, as well as a setup operator type corresponding to the mechanic category and provides support whenever failure of a mechanic, electric or physical, among others, occurs. All these categories or operator types have varying numbers of workers who go on different shifts. For example, in a company like that herein studied that has 21 machines, there could be two qualified technicians, four auxiliary technicians, and one mechanical technician per shift.

The formulation of the MILP model for lot-sizing and scheduling on parallel flexible injection machines with setup common operators is described next. The objective function minimizes total costs, which include setup costs with common setup operators, inventory costs, penalization costs for coverage stockouts, backorder costs, and tool setup costs, such as the route cost of selecting one machine or another to set up a tool.

$$
\operatorname{Min} z=\sum_{i} \sum_{l} \sum_{j} \sum_{t} c s_{j} \cdot S A_{i l j t}+\sum_{i} \sum_{l} \sum_{j} \sum_{t} s c_{i l j t} \cdot S A_{i l j t}+\sum_{k} \sum_{t} c i_{k} \cdot \mathrm{INV}_{k t}+\sum_{k} \sum_{t} c s t_{k} \cdot S T_{k t}+\sum_{k} \sum_{t} c b_{k} \cdot B_{i t}+\sum_{i} \sum_{l} \sum_{j} \sum_{t} r_{i j} \cdot c r_{i j} \cdot S A_{i l j t},
$$

subject to sequence constraints: 
TABLE 3: Nomenclature for the model.

\begin{tabular}{|c|c|}
\hline Index & \\
\hline$l$ & Index setup type operators $l \in\{1, \ldots, L\}$ \\
\hline & Data \\
\hline$s l a_{i l j}$ & Number of setup type operators $l$ required to setup the tool $j$ on machine $i$ \\
\hline$s c l_{i l j}$ & Cost of type operator $l$ to setup the tool $j$ on machine $i$ \\
\hline$s l_{s l}$ & Number of available workers of type operator $l$ available \\
\hline & Decision variables \\
\hline$S_{i l j t}$ & 1 if the tool $j$ is setup by setup operator $l$ on machine $i$ during time period $t, 0$ otherwise \\
\hline$S A_{i l j t}$ & $\begin{array}{l}1 \text { if tool } j \text { is set up by setup operator } l \text { on machine } i \text { during time period } t \text { and is not set up on machine } i \text { during time period } t-1 ; 0 \text { if } \\
\text { tool } j \text { is set up by setup operator } l \text { on machine } i \text { during time period } t-1\end{array}$ \\
\hline
\end{tabular}

$$
\begin{aligned}
& S_{i l j t} \leq r_{i j}, \quad \forall i, l, j, t, \\
& S A_{i l j t} \leq r_{i j}, \quad \forall i, l, j, t, \\
& \sum_{j} S_{i l j t} \cdot r_{i j} \leq 1, \quad \forall i, l, t, \\
& \sum_{i} S_{i l j t} \cdot r_{i j} \leq a_{j}, \quad \forall j, t, l .
\end{aligned}
$$

Equations (21) and (22) constrain only setup tools $j$ by setup operators $l$ on specific previously assigned machines $m$. Constraint (23) determines that one or any tool $j$ can be set up by setup operator $l$ for production during each time period $t$. Constraint (24) guarantees that the total amount of tools $j$ available can only be set up for production as a maximum during each time period $t$ by setup operator $l$.

Production and capacity constraints:

$$
\begin{aligned}
X n p_{k t}< & =\sum_{i} \sum_{l} \sum_{j} n p_{j k} \cdot r_{i j} \cdot S A_{i l j k}, \quad \forall k, t, \\
T P_{i j t} & =t p_{t} \cdot S_{i l j t}, \quad \forall i, l, j, t .
\end{aligned}
$$

Constraint (25) determines the amount of products $k$ no longer produced when tool $j$ is set up by operator $l$ on machine $I$ during time period $t$ by considering that another tool $j$ is set up on machine $i$ during time period $t-1$. It also ensures that such a specific tool $j$ can be set up on machine $i$ during time period $t$ when product $k$ is produced. Constraint (26) determines the production time spent during time period $t$ when tool $j$ is set up by operator $l$ on machine $i$.

Setup constraints:

$$
\begin{aligned}
S A_{i l j t} & =S_{i l j t}, \quad \forall i, l, j, t=1, \\
S A_{i l j t} & \geq S_{i l j t}-S_{i l j t-1}, \quad \forall i, l, j, t>1, \\
S A_{i l j t} & \leq 1, \quad \forall i, l, j, t>1, \\
\sum_{i} \sum_{j} S A_{i l j t} & \leq n c_{t}, \quad \forall l, t .
\end{aligned}
$$

Constraint (27) allows the first setup of tool $j$ performed by operator $l$ on machine $i$ to be determined and enables it to be modeled if tool $j$ is set up during time period $t$ on machine $i$ for the first time. Decision variables $S_{i l j t}$ and $S A_{i l j t}$ take the same value 1 . Constraint (28) ensures that $S A_{i l j t}$ does not take values above 1 . Constraint (29) limits the amount of tool $j$ changes allowed during time period $t$ and set up by operator $l$ on machine $i$.

Labor constraint:

$$
\sum_{i} \sum_{j} S A_{i l j t} \cdot s l a_{i l j} \leq s l s_{l}, \quad \forall l, t
$$

Constraint (30) limits the amount of tool changes allowed during time period $t$ to the available number of workers of type operator $l$ by considering the number of setup type operators $l$ required to set up tool $j$ on machine $i$.

Bound and nature variables:

$$
S A_{i l j t}, S_{i l j t} \in\{0,1\}, \quad \forall i, l, j, t .
$$

Constraint (31) indicates the binary nature of the setup $S_{i l j t}$ and setup amount $S A_{i l j t}$ variables. Finally, the MILP model for lot-sizing and scheduling on parallel flexible injection machines with setup common operators is also subject to constraints (6), (8), (13a), (13b), (14)-(16), (18), and (19).

\section{Case Study and Computational Experiments}

The proposed base MILP model for lot-sizing and scheduling on parallel flexible injection machines and the extended MILP model for lot-sizing and scheduling on parallel flexible injection machines with setup common operators were implemented in Python 3.8.2, using Pyomo [9] as an extensible python-based open-source optimization modeling language for linear programming. The performance of the proposed model was evaluated on a set of instances that reflect different characteristics of the real-world case of the automotive components industry under study. All the numerical tests were conducted on a personal computer equipped with an Intel (R) Core (TM) I5-8500@ $3.00 \mathrm{GHz}$ Processor and 8 GB RAM. We used Python 3.8.2 and tested applying Gurobi 9.0 to solve the mixed integer linear programming model.

In the next section of data generation, it is described how the data are generated to run the computational experiments. The datasets generated to validate the proposed models correspond to small, medium, and large datasets. Sized datasets can be accessed through a link available at the end of the document. Finally, the last section presents the results of the computational experiments carried out. 
6.1. Data Generation. For the data generation, we define various instance sets, including small, medium, and large data sizes. The small dataset corresponds to the minimum amount of data required to test the proposed model; the medium dataset allows to test the model with a reasonable number of parameters and variables to be solved by the model, approaching to the realistic view of the LSSP; and finally, the large dataset replicates the real amount of data managed by real-world enterprises when solving the LSSP. For the computational experiments, all the datasets are built through considering the parameter values depicted in $\mathrm{Ta}-$ ble 5. The data values are created in the way that mostly represents real data from the automotive components industry; next, the data values are defined as follows:

The parameter $a_{j}$ determines the total amount of tools $j$ available for production; in this regard, only one unit of each tool is available, and this means that there are not duplicated tools to produce the same components.

The backorder cost $\left(c b_{k}\right)$ and the coverage stockout cost $\left(c s t_{k}\right)$ are represented by a very high value (equal to $M$ (99999)) in order to avoid customer missing parts in the model resolution.

Inventory costs $\left(c i_{k}\right)$ are set with values uniformly distributed in given interval; $U(u 1, u 2)$ is a random variable which is uniformly distributed on $\left[u_{1}, u_{2}\right]$.

The stock coverage is defined in three days of demand. Nevertheless, in small datasets where the number of periods is lower than three, we have considered one coverage day of demand $\left(\operatorname{cov}_{k t}=1\right)$ in order not to have unfeasible solutions in the model resolution, in such a way that the model considers one period of future demand $(\mathrm{d} t+1)$ to be produced during period $t$.

Random $\left[r_{1}, r_{2}\right]$ values denote a random integer value over the interval from $r_{1}$ to $r_{2}$. The following data parameters use Random $\left[r_{1}, r_{2}\right]$ values: setup cost of a tool $\left(c r_{i j}\right)$, setup cost of preparing a tool $\left(c s_{j}\right)$, maximum inventory (INVMAXk), and the amount of products no longer produced when a tool is set up $\left(\mathrm{np}_{j k}\right)$.

The amount of products produced when a tool is set up $\left(p_{j k}\right)$ is also denoted as Random $\left[r_{1}, r_{2}\right]$. In this regard, $\left[r_{1}, r_{2}\right]$ indicates that product $k$ is assigned to the tool $j$; otherwise, $p_{j k}=0$. Parts are randomly assigned to tools, with the condition that each part must be assigned to one tool. Table 4 proposes an example of assignment on a small dataset composed of three tools and six products.

In order to generate the values for the demand, blocks of seven time periods corresponding to the 7 days of the week are considered. In this regard, the first five periods of the week will have demand values set as Random (15, 40); otherwise, $d_{k t}=0$ on the sixth and seventh periods of the week, that is, on Saturday and Sunday.

The minimum inventory (INVMINk) is set as one unit for all the products $k$; accordingly, the initial inventory $\left(\mathrm{INV}_{k 0}\right)$ is also set as one unit for all products $k$.
The amount of tool changes allowed $(n c t)$ is defined by Random $(I, I+5)$ with $I$ being the minimum number of machines changes allowed, which coincides with the total number of machines. Considering the same scheme of blocks of weeks divided in 7 periods, no tool changes are allowed in the $7^{\text {th }}$ period of the week, $n c t=0$; this is because on Sundays the enterprise does not produce, uses it as a day of rest or for machine maintenance, etc.

According to this last statement, the time available for production $(t p t))$ is 24 hours for the first five periods of the week and 16 hours for the $6^{\text {th }}$ period of the week. No time production is available for the $7^{\text {th }}$ period of the week.

$r_{i j}=1$ indicates that all the tools can be set up on all the machines.

The aforementioned parameters and values are defined for the base model. The three parameters added for the extended model, which considers setup common operators, are described in Table 5.

$s l_{\text {ail } j}=1$ indicates that one setup type operator is required to setup the tool $j$ on machine $i$

The cost of type operator $l$ to setup the tool $j$ on machine $i\left(s c l_{i l j}\right)$ is set with values uniformly distributed in given interval; $U\left(u_{1}, u_{2}\right)$ is a random variable which is uniformly distributed on $\left[u_{1}, u_{2}\right]$.

Finally, the number of available workers of type operator $l$ available $\left(s s_{l}\right)$ must be as much as the number of machines $I$.

In addition, we attach the link to the synthetic data generator, so that the model can be reproduced in future research http://hdl.handle.net/10251/161636.

6.2. Results and Computational Experiments. Using the aforementioned synthetic data generator, a set of experiments have been conducted to validate the two proposed models: (i) base LSSP model, an MILP model for lot-sizing and scheduling on parallel flexible injection machines, and (ii) extended LSSP model, an MILP model for lot-sizing and scheduling on parallel flexible injection machines with setup common operators.

In order to give the reader a clear insight of the input data parameter values and the output data results once implemented the proposed MILP, we include an example of a small dataset size. The small dataset of the base model is characterized by having 2 machines, 4 tools, 6 parts, and 3 periods. The results obtained with the decision variables in the MILP model for lot-sizing and scheduling on parallel flexible injection machines are presented in Tables 6 and 7.

According to the results obtained in the MILP model for lot-sizing and scheduling on parallel flexible injection machines, all the available capacity is occupied in the three defined periods. Notwithstanding, demand was higher than the available capacity and, therefore, the model had to delay the demand of products $k=2,3,4$. Figure 3 shows the Gantt chart showing the schedule obtained after applying the base 
TABLe 4: Amount of products produced when a tool is set up.

\begin{tabular}{lll}
\hline$j$ & $k$ & $p_{j k}$ \\
\hline 1 & 4 & 4 \\
2 & 1 & 2 \\
2 & 5 & 2 \\
2 & 6 & 2 \\
3 & 2 & 2 \\
4 & 3 & 3 \\
\hline
\end{tabular}

TABLE 5: Generation of values for data parameters.

\begin{tabular}{|c|c|}
\hline Parameter & Value \\
\hline$a_{j}$ & 1 \\
\hline$c b_{k}$ & 99999 \\
\hline$c i_{k}$ & $U(0.1,1)$ \\
\hline $\operatorname{cov}_{k t}$ & 1 when $T<3$; otherwise, 3 when $T>3$ \\
\hline$c r_{i j}$ & Random $(5,15)$ \\
\hline$c s_{j}$ & Random $(45,50)$ \\
\hline cst $_{k}$ & 99999 \\
\hline$d_{k t}$ & Random $(15,40)$ if $T=$ first 5 periods of the week; otherwise, 0 if $T=6^{\text {th }}$ and $T=7^{\text {th }}$ periods of the week \\
\hline $\mathrm{INV}_{k 0}$ & 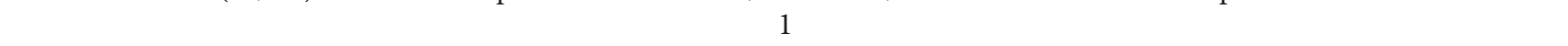 \\
\hline INVMAX $_{k}$ & Random $(10000,20000)$ \\
\hline INVMIN $_{k}$ & 1 \\
\hline$n c_{t}$ & Random $(I, I+5) 0$ if $T=7^{\text {th }}$ period of the week \\
\hline$n p_{j k}$ & Random $(2,5)$ \\
\hline$p_{j k}$ & Random $(2,5)$ \\
\hline$t p_{t}$ & 24 hours if $T=$ first 5 periods of the week; 16 hours if $T=6^{\text {th }}$ period of the week; 0 hours if $T=7^{\text {th }}$ period of the week \\
\hline$r_{i j}$ & $x_{0}$ \\
\hline$s l a_{i l j}$ & 1 \\
\hline$s c l_{i l j}$ & $U(2.5,3.5)$ \\
\hline$s s_{l}$ & $I$ \\
\hline
\end{tabular}

TABLE 6: Small dataset results of the base LSSP MILP: lot-sizing, inventories, coverage stockout, and backorders.

\begin{tabular}{cccccccc}
\hline$k$ & $t$ & $X_{k t}$ & $X n p_{k t}$ & $X_{k t}^{\prime}$ & $S T_{k t}$ & $\mathrm{INV}_{k t}$ & $B_{k t}$ \\
\hline 1 & 1 & 48 & 0 & 48 & 2 & 21 & 0 \\
1 & 2 & 27 & 0 & 27 & 0 & 25 & 0 \\
1 & 3 & 0 & 0 & 0 & 0 & 1 & 0 \\
2 & 1 & 0 & 0 & 0 & 33 & 1 & 35 \\
2 & 2 & 48 & 0 & 48 & 30 & 1 & 21 \\
2 & 3 & 48 & 0 & 48 & 0 & 1 & 4 \\
3 & 1 & 72 & 0 & 72 & 0 & 39 & 0 \\
3 & 2 & 0 & 0 & 0 & 11 & 19 & 0 \\
3 & 3 & 0 & 0 & 0 & 0 & 1 & 12 \\
4 & 1 & 0 & 0 & 0 & 14 & 1 & 30 \\
4 & 2 & 0 & 0 & 0 & 32 & 1 & 45 \\
4 & 3 & 78 & 0 & 78 & 0 & 1 & 0 \\
5 & 1 & 48 & 0 & 48 & 8 & 31 & 0 \\
5 & 2 & 41 & 0 & 41 & 0 & 33 & 0 \\
5 & 3 & 0 & 0 & 0 & 0 & 1 & 0 \\
6 & 1 & 48 & 0 & 48 & 19 & 15 & 0 \\
6 & 2 & 48 & 0 & 48 & 5 & 29 & 0 \\
6 & 3 & 0 & 0 & 0 & 0 & 1 & 6 \\
\hline
\end{tabular}

MILP model. Each row represents a machine $i$ and each rectangle a tool $j$; inside the rectangle, we have indicated the parts $k$ and processing time $t$ of each tool $j$.
TABLE 7: Small dataset results of the base LSSP MILP: scheduling.

\begin{tabular}{cccccc}
\hline$i$ & $j$ & $t$ & $S A_{i j t}$ & $S_{i j t}$ & $T P_{i j t}$ \\
\hline 1 & 2 & 1 & 1 & 1 & 24 \\
1 & 2 & 2 & 0 & 1 & 24 \\
1 & 1 & 3 & 1 & 1 & 24 \\
2 & 4 & 1 & 1 & 1 & 24 \\
2 & 3 & 2 & 1 & 1 & 24 \\
2 & 3 & 3 & 0 & 1 & 24 \\
\hline
\end{tabular}

The input and output data for the small, medium, and large datasets of the base LSSP model are presented at https://doi.org/10.4995/Dataset/10251/161642.

The input data of the extended LSSP model are characterized by considering 2 machines, 4 tools, 6 parts, 3 periods, and 4 type operators. The results obtained with the decision variables in the MILP model for lot-sizing and scheduling on parallel flexible injection machines with setup common operators are presented in Tables 8 and 9.

According to the obtained results, all the available capacity is occupied along the three defined periods. Nevertheless, demand was higher than the available capacity and, therefore, the model had to delay the demand of products $k=2,4$ in periods $t=1,2$ and had to delay the demand of products $k=3,6$ in period $t=3$. The solution provided in 


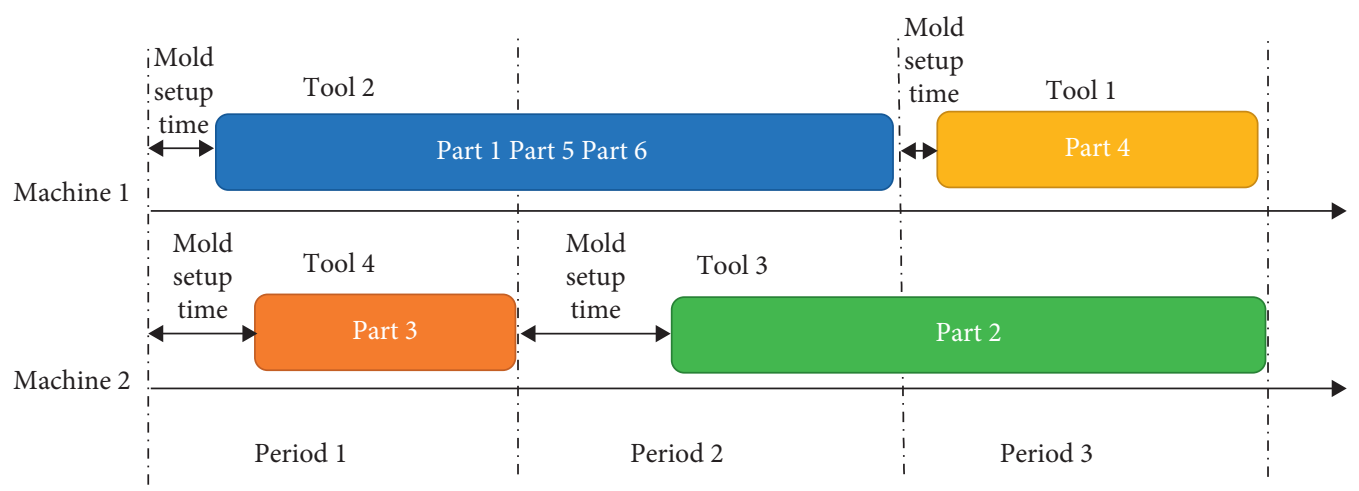

FIgURE 3: Gantt chart base MILP model for lot-sizing and scheduling on parallel flexible injection machines.

TABLE 8: Small dataset results of the extended LSSP MILP, with setup common operators: lot-sizing, inventories, coverage stockout, and backorders.

\begin{tabular}{|c|c|c|c|c|c|c|c|}
\hline$k$ & $t$ & $X_{k t}$ & $X n p_{k t}$ & $X_{k t}^{\prime}$ & $S T_{k t}$ & $\mathrm{INV}_{k t}$ & $B_{k t}$ \\
\hline 1 & 1 & 48 & 0 & 48 & 2 & 21 & 0 \\
\hline 1 & 2 & 27 & 0 & 27 & 0 & 25 & 0 \\
\hline 1 & 3 & 0 & 0 & 0 & 0 & 1 & 0 \\
\hline 2 & 1 & 0 & 0 & 0 & 33 & 1 & 35 \\
\hline 2 & 2 & 48 & 0 & 48 & 30 & 1 & 21 \\
\hline 2 & 3 & 48 & 0 & 48 & 0 & 1 & 4 \\
\hline 3 & 1 & 72 & 0 & 72 & 0 & 39 & 0 \\
\hline 3 & 2 & 0 & 0 & 0 & 11 & 19 & 0 \\
\hline 3 & 3 & 0 & 0 & 0 & 0 & 1 & 12 \\
\hline 4 & 1 & 0 & 0 & 0 & 14 & 1 & 30 \\
\hline 4 & 2 & 0 & 0 & 0 & 32 & 1 & 45 \\
\hline 4 & 3 & 78 & 0 & 78 & 0 & 1 & 0 \\
\hline 5 & 1 & 48 & 0 & 48 & 8 & 31 & 0 \\
\hline 5 & 2 & 41 & 0 & 41 & 0 & 33 & 0 \\
\hline 5 & 3 & 0 & 0 & 0 & 0 & 1 & 0 \\
\hline 6 & 1 & 48 & 0 & 48 & 19 & 15 & 0 \\
\hline 6 & 2 & 48 & 0 & 48 & 5 & 29 & 0 \\
\hline 6 & 3 & 0 & 0 & 0 & 0 & 1 & 6 \\
\hline
\end{tabular}

TABLE 9: Small dataset results of the base LSSP MILP: scheduling.

\begin{tabular}{|c|c|c|c|c|c|c|}
\hline$i$ & $l$ & $j$ & $t$ & $S_{i l j t}$ & $S A_{i l j t}$ & $T P_{i j t}$ \\
\hline 1 & 1 & 2 & 1 & 1 & 1 & 24 \\
\hline 1 & 2 & 2 & 1 & 1 & 1 & 0 \\
\hline 2 & 1 & 4 & 1 & 1 & 1 & 0 \\
\hline 2 & 2 & 4 & 1 & 1 & 1 & 0 \\
\hline 1 & 2 & 4 & 1 & 0 & 0 & 24 \\
\hline 2 & 1 & 3 & 2 & 1 & 1 & 0 \\
\hline 2 & 2 & 3 & 2 & 1 & 1 & 0 \\
\hline 1 & 1 & 2 & 2 & 1 & 0 & 24 \\
\hline 1 & 2 & 2 & 2 & 1 & 0 & 0 \\
\hline 1 & 2 & 3 & 2 & 0 & 0 & 24 \\
\hline 1 & 1 & 1 & 3 & 1 & 1 & 24 \\
\hline 1 & 2 & 1 & 3 & 1 & 1 & 0 \\
\hline 2 & 1 & 3 & 3 & 1 & 0 & 0 \\
\hline 2 & 2 & 3 & 3 & 1 & 0 & 0 \\
\hline 1 & 2 & 3 & 3 & 0 & 0 & 24 \\
\hline
\end{tabular}




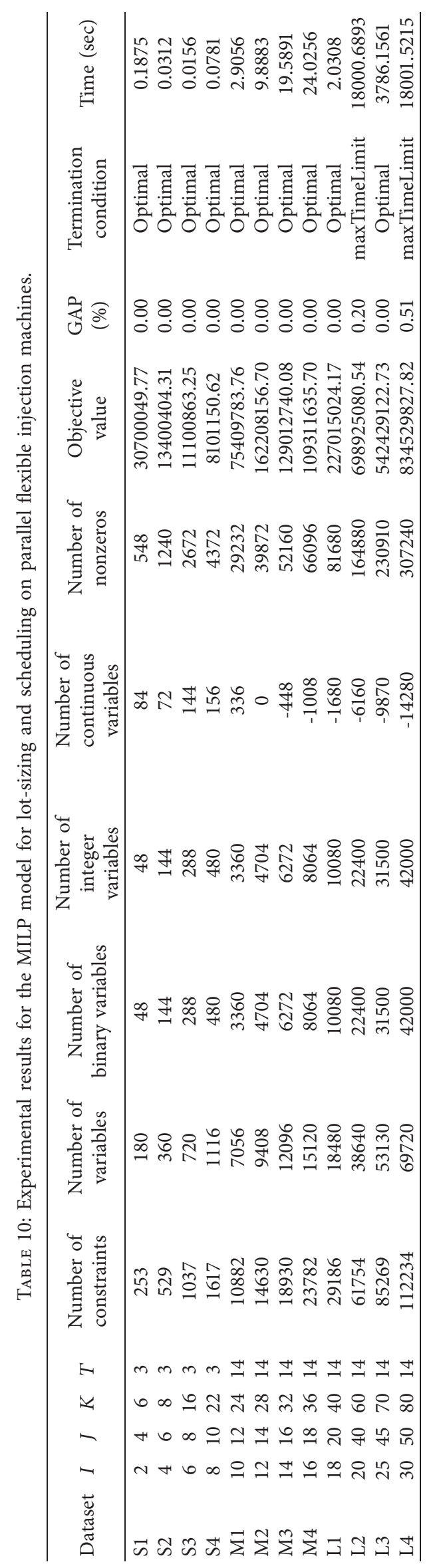




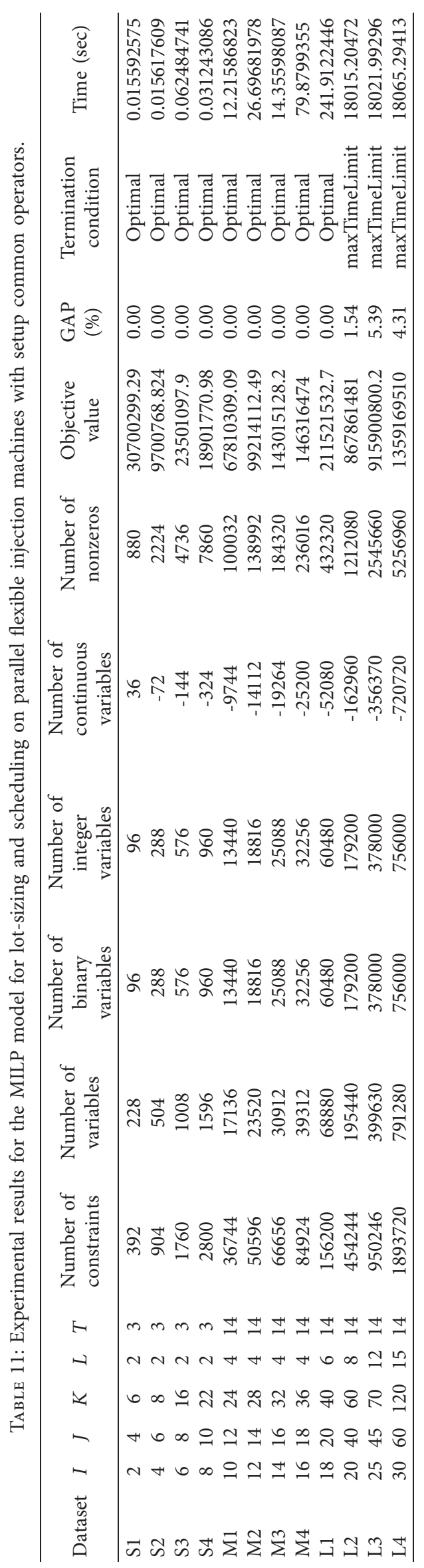


the scheduling shows that the two type operators $l$ are assigned to change the tools $j$.

The experimental results obtained with the tests conducted for all the datasets employed in the validation for both the base model and the extended model in which the labor index is added are presented in Tables 10 and 11. The input and output data for the small, medium, and large datasets of the extended LSSP model are presented at https://doi.org/10.4995/Dataset/10251/161643. Four instances are generated of each dataset size. The synthetic instances for the base model have been generated through the aforementioned proposed generator: http://hdl. handle.net/10251/161636. The synthetic instances for the extended model with common setup operators have been generated through the following generator: http:// hdl.handle.net/10251/161635.

The computational results (Table 10) show that the MILP model for lot-sizing and scheduling on parallel flexible injection machines in small (S1, S2, S3, and S4) and medium (M1, M2, M3, and M4) instances achieves optimal results $(\mathrm{GAP}=0 \%)$ in, at maximum, 24 seconds. With regard to the large instances generated (L1, L2, L3, and L4), the calculation time on average is set as 2.8 hours, finding a very near-optimal solution, with an average $0.24 \%$ GAP. In this regard, it is worth to highlight that the large dataset L1 achieves the optimal solution in a very reduced computational time of 2 seconds.

The computational results (Table 11) show that the MILP model for lot-sizing and scheduling on parallel flexible injection machines with setup common operators in small (S1, S2, S3, and S4) and medium (M1, M2, M3, and M4) instances achieves optimal results $(\mathrm{GAP}=0 \%)$ in, at maximum, 79.9 seconds. With regard to the large instances generated (L1, L2, $\mathrm{L} 3$, and L4), the calculation time on average is set as 3.7 hours finding a very near-optimal solution, with an average $2.81 \%$ GAP. In this regard, it is worth to highlight that the large dataset L1 achieves the optimal solution in a very reduced computational time of 242 seconds.

The experiments carried out for both the base model and the extended model that consider common setup operators are valid for its application on solving LSSP models with real amount of data managed by real-world enterprises.

\section{Discussion and Conclusions}

This paper addresses the LSSP applied to an automotive plastic components' enterprise. An MILP base model is proposed to deal with the lot-sizing and scheduling problem on parallel flexible injection machines to mainly minimize the setup, inventory, stockout, and backorder costs by taking into account injection molds as the main index to schedule parallel flexible injection machines. The MILP base model is extended to provide the enterprise under study with a more realistic solution that considers setup common operators. Therefore, the extended LSSP model, an MILP model for lotsizing and scheduling on parallel flexible injection machines with setup common operators, is presented. This produces a model that adapts to the restrictions of the company under study, an automotive plastic components' enterprise. The peculiarity of this model is that it takes injection molds as the main index to schedule parallel flexible injection machines by considering setup common operators. The novelty of both proposed models lies in our study considering many periods when modeling and running experiments to solve the LSSP. Moreover, the proposed MILP bears in mind stock coverage constraints, which are typical in the studied automotive supply chain industry context, and contemplates an objective function that allows idle times among molds, which is a fundamental characteristic for real cases and has been ignored by former studies.

Finally, this paper validates the proposed MILP by performing experiments with different sized instances, including small, medium, and large datasets. The large dataset is characterized by replicating the amount of data used in the real enterprise that is the object of this study. The goodness of the model is evaluated with the computational time and the deviation of the obtained results as regards the optimal solution.

This study is not without its limitations. The small and medium datasets are solved in both cases in very efficient computing times. The application of the proposed model using the large dataset is more limited in computational efficiency terms. To solve this problem, the literature indicates the generation of heuristics, metaheuristics, and matheuristics. The last type falls within the authors' future research options as far as the contemplated model herein is concerned. Thus, the first research line intends to improve the computational efficiency to solve the model by applying matheuristics, which would consist in solving the binary variables in both the base model $\left(S_{i j t}\right.$ and $\left.S A_{i j t}\right)$ and its extended version $\left(S_{i l j t}\right.$ and $\left.S A_{i l i t}\right)$ by a metaheuristic technique, e.g., genetic algorithms, taboo search, and simulated annealing. The metaheuristics result will be provided as input data for the MILP. The following research lines focus on improving the model by considering new constraints that will be very useful for the real firm. In this way, the second future research line is a second extension of the base model that bears in mind the availability of materials. For this purpose, inventory equations are to be added that consider the list of materials needed to manufacture end products. Finally, for the third future research line, a third extension of the model is proposed by considering space limitations in a warehouse's volume. In this way, part volumes are calculated to meet both stock coverage and limited warehouse space, contemplated from the premise that all parts have different volume requirements. This means that the model and its third extension (or third generation) will be capable of meeting the stock coverage of large-sized parts and stocking small-sized ones so that when they have to be produced, the production resources related to bigger pieces will be used. The third-generation model will allow bigger-sized parts to be stored, which will be left at the stock coverage level by calculating the quantity of smaller parts whose coverage can be extended.

\section{Data Availability}

The instances generated (input and output datasets) and analyzed during the study are available at the following links: (i) base LSSP model, an MILP model for lotsizing and scheduling on parallel flexible injection machines: https://doi.org/10.4995/Dataset/10251/161642; 
(ii) extended LSSP model, an MILP model for lot-sizing and scheduling on parallel flexible injection machines with setup common operators: https://doi.org/10.4995/ Dataset/10251/161643. The algorithms developed for generating the synthetic datasets are available at the following links: (i) base LSSP model, an MILP model for lot-sizing and scheduling on parallel flexible injection machines: http://hdl.handle.net/10251/161636; (ii) extended LSSP model, an MILP model for lot-sizing and scheduling on parallel flexible injection machines with setup common operators: http://hdl.handle.net/10251/ 161635.

\section{Conflicts of Interest}

The authors declare that there are no conflicts of interest regarding the publication of this study.

\section{References}

[1] R. Gujjula, S. Werk, and H.-O. Günther, A heuristic based on Vogel's approximation method for sequencing mixed-model assembly lines," International Journal of Production Research, vol. 49, no. 21, pp. 6451-6468, 2011.10.1080/00207543.2010.527384

[2] S.-i. Kim, J. Han, Y. Lee, and E. Park, "Decomposition based heuristic algorithm for lot-sizing and scheduling problem treating time horizon as a continuum," Computers \& Operations Research, vol. 37, no. 2, pp. 302-314, 2010.10.1016/j.cor.2009.05.007

[3] H. Lasi, P. Fettke, H.-G. Kemper, T. Feld, and M. Hoffmann, "Industry 4.0," Business \& Information Systems Engineering, vol. 6, no. 4, pp. 239-242, 2014.10.1007/s12599-014-0334-4

[4] S. Echchakoui and N. Barka, "Industry 4.0 and its impact in plastics industry: a literature review," Journal of Industrial Information Integration, vol. 20, no. p. 100172, 2020.10.1016/j.jii.2020.100172

[5] H2020 Project C2NET, "'Cloud Collaborative Manufacturing Networks' (C2NET).” 2017.

[6] R. Sanchís, B. Andrés, R. Poler, J. Mula, and M. Díaz-Madroñero, "La solución de optimización C2NET," Dirección y Organización, vol. 64, 64, pp. 36-41, 2018.10.37610/dyo.v0i64.520

[7] K. P. Abdul Nazar and V. Madhusudanan Pillai, "Mixed-model sequencing problem under capacity and machine idle time constraints in JIT production systems," Computers \& Industrial Engineering, vol. 118, pp. 226-236, 2018.10.1016/j.cie.2018.02.032

[8] J. Mula, A. C. Lyons, J. E. Hernández, and R. Poler, “An integer linear programming model to support customerdriven material planning in synchronised, multi-tier supply chains," International Journal of Production Research, vol. 52, no. 14, pp. 4267-4278, Jan. 2014.10.1080/ 00207543.2013.878055

[9] W. E. Hart, C. Laird, J.-P. Watson, and D. L. Woodruff, Pyomo - Optimization Modeling in Python, 1st ed. Springer Publishing Company, Berlin, Germany, 2012

[10] Y. Á. Ríos-Solís, O. J. Ibarra-Rojas, M. Cabo, and E. Possani, "A heuristic based on mathematical programming for a lot-sizing and scheduling problem in mold-injection production," European Journal of Operational Research, vol. 284, no. 3, pp. 861-873, 2020.10.1016/j.ejor.2020.01.016

[11] O. J. Ibarra-Rojas, R. Z. Ríos-Mercado, Y. A. RiosSolis, and M. A. Saucedo-Espinosa, "A decomposition approach for the piece-mold-machine manufacturing problem," International Journal of Production Economics, vol. 134, no. 1, pp. 255-261, 2011.10.1016/ j.ijpe.2011.07.006

[12] Y. Li, Z. Yang, G. Li, D. Zhao, and W. Tian, "Optimal scheduling of an isolated microgrid with battery storage considering load and renewable generation uncertainties," IEEE Transactions on Industrial Electronics, vol. 66, no. 2, pp. 1565-1575, Feb. 2019.10.1109/tie.2018.2840498

[13] L. Guimarães, D. Klabjan, and B. Almada-Lobo, "Modeling lotsizing and scheduling problems with sequence dependent setups," European Journal of Operational Research, vol. 239, no. 3, pp. 644-662, 2014.10.1016/j.ejor.2014.05.018

[14] H. Stadtler, "Multi-level single machine lot-sizing and scheduling with zero lead times," European Journal of Operational Research, vol. 209, no. 3, pp. 241-252, 2011.10.1016/j.ejor.2010.09.022

[15] C. Wolosewicz, S. Dauzère-Pérès, and R. Aggoune, "A Lagrangian heuristic for an integrated lot-sizing and fixed scheduling problem," European Journal of Operational Research, vol. 244, no. 1, pp. 3-12, 2015.10.1016/j.ejor.2015.01.034

[16] R. J. W. James and B. Almada-Lobo, "Single and parallel machine capacitated lotsizing and scheduling: new iterative MIP-based neighborhood search heuristics," Computers \& Operations Research, vol. 38, no. 12, pp. 1816-1825, 2011.10.1016/j.cor.2011.02.005

[17] H. Meyr and M. Mann, "A decomposition approach for the general lotsizing and scheduling problem for parallel production lines," European Journal of Operational Research, vol. 229, no. 3, pp. 718-731, 2013.10.1016/j.ejor.2013.03.036

[18] M. Díaz-Madroñero, J. Mula, B. Andres, R. Poler, and R. Sanchis, "A capacitated lot-sizing and scheduling model for the bi-part injection moulding problem," In Proceeding of the 11th International Conference on Industrial Engineering and Industrial Management (CIO2017),Valencia, Spain July 2017.

\section{Acknowledgments}

This work was supported by the Conselleria de Educación, Investigación, Cultura y Deporte-Generalitat Valenciana for hiring predoctoral research staff with Grant no. ACIF/2018/170 
and European Social Fund with Grant Operational Program of FSE 2014-2020, the Valencian Community, and the authors would like to acknowledge the support of the researchers participating in the collaborative projects "Cloud Collaborative Manufacturing Networks" (C2NET) (http://c2net-project.eu/), which has received funding from the EU Horizon 2020 Research and Innovation Programme with grant agreement no. 63690, and "Zero Defects Manufacturing Platform" (ZDMP) (http://www.zdmp.eu), which has received funding from the EU Horizon 2020 Research and Innovation Programme with grant agreement no. 825631 .

\section{References}

[1] B. Andres, E. Guzman, and R. Poler, "A novel MILP model for the production, lot sizing, and scheduling of automotive plastic components on parallel flexible injection machines with setup common operators," Complexity, vol. 2021, Article ID 6667516, 16 pages, 2021. 